\title{
NUMERICAL RANGE OF A WEIGHTED SHIFT WITH PERIODIC WEIGHTS
}

\author{
WILLIAM C. RIDGE
}

\begin{abstract}
Calculation of the numerical range of a weighted shift is reduced to the solution of a polynomial equation when the weights form a periodic sequence, or approach a periodic sequence from below.
\end{abstract}

Introduction. A weighted shift on $l^{2}$ or $l_{+}^{2}$ is a linear operator $S$ defined by $S e_{n}=s_{n} e_{n+1}$ where $\left\{e_{n}\right\}$ is an orthonormal basis, and $\left\{s_{n}\right\}$ a sequence of complex numbers. The numerical range of an operator $S$ is the set of complex numbers $(S x, x)$ where $\|x\|=1$; this is denoted $W(S)$. For definiteness we assume here a one-sided shift, indexed by positive integers; the proofs and results are the same for a two-sided shift.

Then we are given

$$
\begin{aligned}
x & =x_{1} e_{1}+x_{2} e_{2}+\cdots, \quad x_{i} \text { complex, } \quad \sum\left|x_{i}\right|^{2}<\infty ; \\
S x & =x_{1} s_{1} e_{2}+x_{2} s_{2} e_{3}+\cdots .
\end{aligned}
$$

We begin with some simple facts about weighted shifts [1].

(1) $S$ is a bounded operator if and only if $\left\{s_{n}\right\}$ is a bounded sequence, and then $\|S\|=\sup \left|s_{n}\right|$.

(2) $S$ is unitarily equivalent to a shift with weights $t_{i}$ whenever $\left|t_{i}\right|=\left|s_{i}\right|$ for all $i$. In particular, $S$ is unitarily equivalent to $c S$ whenever $|c|=1$.

(3) Therefore $W(S)$ has circular symmetry about $0: c W=W$ whenever $|c|=1$.

(4) Since $W$ is convex, it follows that $W(S)$ is a disk centered at 0 ; its radius $w(S)$ is the numerical radius of $S$.

It is an easy exercise to find $W(S)$ in some special cases. For example:

(5) If $\left|s_{n}\right| \leqslant K$ for all $n$, and $\left|s_{n}\right| \rightarrow K$, then $W(S)=K$.

By (2) it suffices to consider shifts with real nonnegative weights, $s_{n} \geqslant 0$, and we shall do so.

THEOREM 1. If $\left\{s_{n}\right\}$ is a periodic sequence, of period $r$, then

$$
\begin{aligned}
& w(S)=\max \left\{s_{1} x_{1} x_{2}+s_{2} x_{2} x_{3}+\cdots+s_{r} x_{r} x_{1}:\right. \\
& \left.x_{i} \text { real, } x_{1}^{2}+\cdots+x_{r}^{2}=1\right\},
\end{aligned}
$$

and finding this is equivalent to solving a polynomial equation of degree $r$.

Received by the editors April 8, 1974 and, in revised form, April 18, 1975.

AMS (MOS) subject classifications (1970). Primary 47A 10, 47 B99.

Key words and phrases. Hilbert space, operator, numerical range. 
Proof. First consider a sequence $x$ consisting of the finite sequence of complex numbers $\left\{x_{1}, x_{2}, \ldots, x_{r}\right\}$ repeated $k$ times, with 0 's thereafter. Then

$$
\begin{aligned}
S x & =\left\{0,\left[s_{1} x_{1}, s_{2} x_{2}, \ldots, s_{r} x_{r}\right],(\text { repeated } k \text { times }), 0,0, \ldots\right\}, \\
(S x, x) & =k\left(s_{1} x_{1} x_{2}+s_{2} x_{2} x_{3}+\cdots+s_{r} x_{r} x_{1}\right)-s_{r} x_{r} x_{1}, \\
(x, x) & =k\left(\left|x_{1}\right|^{2}+\left|x_{2}\right|^{2}+\cdots+\left|x_{r}\right|^{2}\right),
\end{aligned}
$$

and for large $k$ we see that $(S x, x) /(x, x)$ can be made arbitrarily close to

$$
\frac{s_{1} x_{1} x_{2}+s_{2} x_{2} x_{3}+\cdots+s_{r} x_{r} x_{1}}{\left|x_{1}\right|^{2}+\left|x_{2}\right|^{2}+\cdots+\left|x_{r}\right|^{2}} \text {. }
$$

Therefore $w(S)$ is at least equal to

$$
\max \left\{\left|s_{1} x_{1} x_{2}+\cdots+s_{r} x_{r} x_{1}\right|: x_{i} \text { complex, }\left|x_{1}\right|^{2}+\cdots+\left|x_{r}\right|^{2}=1\right\} .
$$

By multiplying $x_{k}$ by $e^{i \theta_{k}}$ we may make these components real and nonnegative: this gives the problem:

$$
\begin{aligned}
& \text { Maximize } s_{1} x_{1} x_{2}+s_{2} x_{2} x_{3}+\cdots+s_{r} x_{r} x_{1} \\
& \text { subject to } x_{1}^{2}+\cdots+x_{r}^{2}=1, s_{k}, x_{k} \text { real. }
\end{aligned}
$$

The use of Lagrange multipliers gives the system:

$$
\begin{aligned}
s_{r} x_{r}+s_{1} x_{2} & =\lambda x_{1} \\
s_{1} x_{1}+s_{2} x_{3} & =\lambda x_{2} \\
s_{r-1} x_{r-1}+s_{r} \dot{x}_{1} & =\lambda x_{r} .
\end{aligned}
$$

Elimination of the $x_{i}$ gives a polynomial equation in $\lambda$ of degree $r ; x_{2}, \ldots, x_{r}$ are found by substitution (in terms of $x_{1}$ ), and $x_{1}$ is then found by the relation $x_{1}^{2}+\cdots+x_{r}^{2}=1$.

We now establish that $w(S)$ is no greater than this maximum value of $s_{1} x_{1} x_{2}+\cdots+s_{r} x_{r} x_{1}$.

LEMMA. If $a_{k}, b_{k}$ are nonnegative constants with $b_{k} \neq 0$, then

$$
\frac{a_{1}+a_{2}+\cdots}{b_{1}+b_{2}+\cdots} \leqslant \sup _{k} \frac{a_{k}}{b_{k}}
$$

whenever the left side is defined.

Proof. We first show this for finite sums. If $a / c \geqslant b / d$, then

$$
\frac{a+b}{c+d} \leqslant \frac{a+a d / c}{c+d}=\frac{a}{c}
$$

and the result for finite sums follows by induction. Then

$$
\frac{a_{1}+a_{2}+\cdots+a_{n}}{b_{1}+b_{2}+\cdots+b_{n}} \leqslant \max _{k \leqslant n} \frac{a_{k}}{b_{k}} \leqslant \sup _{k \geqslant 1} \frac{a_{k}}{b_{k}}
$$


and hence the lim sup of the left side satisfies the same inequality; this proves the lemma.

Resuming the proof of Theorem 1: suppose $|x|=1$, and write the components of $x$ as

$$
x=\left\{a_{11} a_{12} \cdots a_{1 r} ; a_{21} a_{22} \cdots a_{2 r} ; \ldots\right\},
$$

$a_{i j}$ complex. Then

$$
\begin{aligned}
& \frac{|(S x, x)|}{(x, x)}=\frac{\left|s_{1} a_{11} a_{12}+\cdots+s_{r} a_{1 r} a_{21}+s_{1} a_{21} a_{22}+\cdots+s_{r} a_{2 r} a_{31}+\cdots\right|}{\left|a_{11}\right|^{2}+\cdots+\left|a_{1 r}\right|^{2}+\left|a_{21}\right|^{2}+\cdots+\left|a_{2 r}\right|^{2}+\cdots} \\
& \quad \leqslant \sup _{k} \frac{\left|s_{1} a_{k 1} a_{k 2}+\cdots+s_{r} a_{k r} a_{(k+1) 1}\right|}{\left|a_{k 1}\right|^{2}+\cdots+\left|a_{k r}\right|^{2}}
\end{aligned}
$$

and

$$
\leqslant \sup _{k} \frac{\left|s_{1} a_{k 1} a_{k 2}+\cdots+s_{r} a_{k r} a_{(k+1) r}\right|}{\left|a_{(k+1) 1}\right|^{2}+\left|a_{k 2}\right|^{2}+\cdots+\left|a_{k r}\right|^{2}} \text {. }
$$

Inequality (1) follows from the lemma, and (2) follows by deleting $\left|a_{11}\right|^{2}$ from the denominator (thus increasing the value of the fraction), regrouping terms of the denominator, and applying the lemma.

Setting

$$
x_{1}=\max \left(\left|a_{k 1}\right|,\left|a_{(k+1) 1}\right|\right), \quad x_{j}=\left|a_{k j}\right| \quad \text { for } j=2, \ldots, r,
$$

we see that

$$
\frac{|(S x, x)|}{(x, x)} \leqslant \max \left\{s_{1} x_{1} x_{2}+\cdots+s_{r} x_{r} x_{1}: x_{1}^{2}+\cdots+x_{r}^{2}=1, x_{i} \text { real }\right\},
$$

and therefore $w(S)$ is equal to this maximum value; this completes the proof of Theorem 1.

THEOREM 2. If $s_{k} \leqslant p_{k}$ and $s_{k}-p_{k} \rightarrow 0$ as $k \rightarrow \infty$, where $\left\{p_{k}\right\}$ is a periodic sequence with period $r$, then

$$
w(S)=\max \left\{p_{1} x_{1} x_{2}+\cdots+p_{r} x_{r} x_{1}: x_{1}^{2}+\cdots+x_{r}^{2}=1, x_{i} \text { real }\right\} .
$$

Proof. Given $\varepsilon>0$, letting $T$ be the shift with weights $p_{k}$, let $x$ be a unit vector such that $(T x, x)>w(T)-\varepsilon$. Choose $n$ such that $\left|s_{k}-p_{k}\right|<\varepsilon$ for $k \geqslant n$. Let $y$ be the unit vector with $y_{k}=0, k=1, \ldots, n ; y_{k+n}=x_{k}, k$ $=1,2, \ldots$ Then $(T y, y)=(T x, x)>w(T)-\varepsilon$.

Now

$$
\|T y-S y\| \leqslant \sup _{k>n}\left|p_{k}-s_{k}\right| \leqslant \varepsilon
$$

so $|(T y, y)-(S y, y)|<\varepsilon$ and so $(S y, y)>w(T)-2 \varepsilon$.

Therefore $w(S) \geqslant w(T)$. Since $0 \leqslant s_{k} \leqslant p_{k}$, we easily have $w(S) \leqslant w(T)$, and so the two are equal. By Theorem 1 ,

$w(T)=\max \left\{p_{1} x_{1} x_{2}+\cdots+p_{r} x_{r} x_{1}: x_{1}^{2}+\cdots+x_{r}^{2}=1, x_{i}\right.$ real $\}=w(S) ;$

this proves Theorem 2 . 
EXAMPLES. (1) If $r=2$ the weights are $a, b, a, b, \ldots$; we are to maximize $(a+b) x_{1} x_{2}$, that is, maximize $x_{1} x_{2}$ subject to $x_{1}^{2}+x_{2}^{2}=1$. The solution is $x_{1}=x_{2}=1 / \sqrt{2},(a+b) x_{1} x_{2}=(a+b) / 2$, and so the numerical radius is the average of the two weights.

(2) If $r=3$ the weights are $a, b, c, a, b, c, \ldots$; we must maximize $a x_{1} x_{2}$ $+b x_{2} x_{3}+c x_{3} x_{1}$ with $x_{1}^{2}+\cdots+x_{3}^{2}=1$; we have the system

$$
a x_{2}+c x_{3}=\lambda x_{1}, \quad a x_{1}+b x_{3}=\lambda x_{2}, \quad b x_{2}+c x_{1}=\lambda x_{3},
$$

which (if $x_{1} \neq 0$ ) leads to the cubic equation $\lambda^{3}-\left(a^{2}+b^{2}+c^{2}\right) \lambda-2 a b c$ $=0$.

Notes. (1) The numerical range is always a disk about 0 , of positive radius except in the trivial case where all the weights are zero.

(2) If any weight is zero then the disk is closed; for (asșuming $s_{r}=0$ for example) then $(S x, x) /(x, x)$ is actually equal to the expression (1), which in turn attains its maximum on the compact sphere (2).

(3) For weights $(1,1,1, \ldots)$ the disk is open; for $|(S x, x)|=1,|x|=1$, would imply $S x=k x,|k|=1$, which is impossible.

(4) I surmise, but have yet to prove, that the disk is open whenever all the weights are nonzero; that is, $(S x, x)$ cannot attain its $\sup w(S)$ for $|x|=1$.

\section{REFERENCE}

1. W. C. Ridge, Approximate point spectrum of a weighted shift, Trans. Amer. Math. Soc. 147 (1970), 349-356. MR 40 \#7843.

DEPARTMENT OF MATHEMATICS, INDIANA-PURDUE UNIVERSITY AT INDIANAPOLIS, INDIANAPOLIS, INDIANA 46205 